\title{
Sky confusion noise in the far-infrared: Cirrus, galaxies and the cosmic far-infrared background ${ }^{\star}$
}

\author{
Cs. Kiss ${ }^{1,2}$, P. Ábrahám ${ }^{1,2}$, U. Klaas ${ }^{1}$, M. Juvela ${ }^{3}$, and D. Lemke ${ }^{1}$ \\ 1 Max-Planck-Institut für Astronomie, Königstuhl 17, 69117 Heidelberg, Germany \\ 2 Konkoly Observatory of the Hungarian Academy of Sciences, PO Box 67, 1525 Budapest, Hungary \\ 3 Helsinki University Observatory, Tähtitorninmäki, PO Box 14, 00014 University of Helsinki, Helsinki
}

Received 31 January 2001 / Accepted 26 September 2001

\begin{abstract}
We examined the sky confusion noise in 40 sky regions by analysing 175 far-infrared $(90-200 \mu \mathrm{m})$ maps obtained with ISOPHOT, the photometer on-board the Infrared Space Observatory. For cirrus fields with $\langle B\rangle>5 \mathrm{MJy} \mathrm{sr}^{-1}$ the formula based on IRAS data (Helou \& Beichman 1990) predicts confusion noise values within a factor of 2 of our measurements. The dependence of the sky confusion noise on the surface brightness was determined for the wavelength range $90 \leq \lambda \leq 200 \mu \mathrm{m}$. We verified that the confusion noise scales as $N \propto\langle B\rangle^{1.5}$, independent of the wavelength and confirmed $N \propto \lambda^{2.5}$ for $\lambda \geq 100 \mu \mathrm{m}$. The scaling of the noise value at different separations between target and reference positions was investigated for the first time, providing a practical formula. Since our results confirm the applicability of the Helou \& Beichman (1990) formula, the cirrus confusion noise predictions made for future space missions with telescopes of a similar size can be trusted. At 90 and $170 \mu \mathrm{m}$ a noise term with a Poissonian spatial distribution was detected in the faintest fields $\left(\langle B\rangle \leq 3-5 \mathrm{MJy} \mathrm{sr}^{-1}\right)$, which we interpret as fluctuations in the Cosmic Far-Infrared Background (CFIRB). Applying ratios of the fluctuation amplitude to the absolute level of $10 \%$ and $7 \%$ at 90 and $170 \mu \mathrm{m}$, respectively, as supported by model calculations, we achieved a new simultaneous determination of the fluctuation amplitudes and the surface brightness of the CFIRB. The fluctuation amplitudes are $7 \pm 2 \mathrm{mJy}$ and $15 \pm 4 \mathrm{mJy}$ at 90 and $170 \mu \mathrm{m}$, respectively. We obtained a CFIRB surface brightness of $B_{0}=0.8 \pm 0.2 \mathrm{MJy} \mathrm{sr}^{-1}\left(\nu I_{\nu}=14 \pm 3 \mathrm{nW} \mathrm{m}^{-2} \mathrm{sr}^{-1}\right)$ at $170 \mu \mathrm{m}$ and an upper limit of $1.1 \mathrm{MJy} \mathrm{sr}^{-1}\left(\nu I_{\nu}=37 \mathrm{nW} \mathrm{m}^{-2} \mathrm{sr}^{-1}\right)$ at $90 \mu \mathrm{m}$.
\end{abstract}

Key words. methods: observational - ISM: structure - infrared: ISM: continuum

\section{Introduction}

Sky confusion noise causes an uncertainty in the determination of the source flux, due to the variation of the sky brightness between the target (on-source) and reference (off-source) positions. This noise cannot be overcome by longer integration times, thus it constitutes a basic limitation for the detection of very faint point sources.

At far-infrared wavelengths the two major components responsible for the sky confusion noise are dust emission from irregularly shaped interstellar clouds, the "galactic cirrus" (Low et al. 1984), and a component of the Extragalactic Background built up from the accumulated light of faint unresolved galaxies along the line of sight (Guiderdoni et al. 1997).

Send offprint requests to: Cs. Kiss, e-mail: pkisscs@konkoly.hu

* Based on observations with ISO, an ESA project with instruments funded by ESA Member States (especially the PI countries: France, Germany, The Netherlands and the UK) and with the participation of ISAS and NASA.
The confusion noise due to cirrus can be characterized by a formalism applied first by Gautier et al. (1992) for the IRAS $100 \mu \mathrm{m}$ scans. They computed the second order structure function for a far-infrared map:

$S(\theta)=\left\langle\left|B(x)-\frac{B(x-\theta)+B(x+\theta)}{2}\right|^{2}\right\rangle_{x}$

where $B$ is the sky brightness, $x$ is the location of the target, $\theta$ is the separation between the target and reference sky positions and the average is taken in spatial coordinates over the whole map. The noise due to sky brightness fluctuations, $N$, is defined as:

$N(\theta)=\sqrt{S(\theta)} \times \Omega$

where $\Omega$ is the solid angle of the measuring aperture.

The measured confusion noise depends both on the size of the measuring aperture $D$ and on the angular separation $\theta$ between the on-source and off-source positions. However, if the sky brightness distribution does not show any characteristic scale length (e.g. random fluctuations 


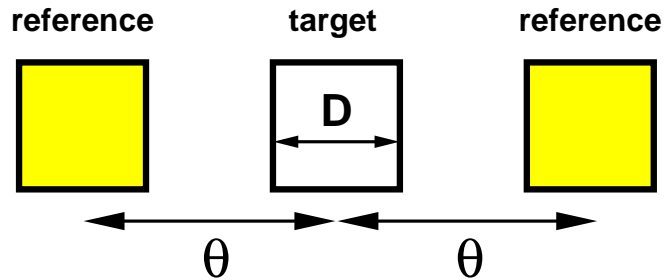

Fig. 1. Measurement configuration to compute the structure noise. Gautier et al. (1992) and Helou \& Beichman (1990) derived their results for the resolution limit $\theta_{\min }=2 D$. In Sect. 3.3 we also consider the cases of $\theta=k \cdot D, k=2 \ldots 7$.

or fractal structure), the only important factor is the $\theta / D$ ratio. For their study of the IRAS $100 \mu \mathrm{m}$ maps Gautier et al. (1992) defined a standard measurement configuration where the on-source position is bracketed by two offsource positions separated by $\pm \theta$ (Fig. 1) and the ratio is fixed to the resolution (Nyquist) limit $\theta=\theta_{\min }=2 D$.

Gautier et al. (1992) pointed out that the Fourier power spectra of the IRAS $100 \mu \mathrm{m}$ scans were generally well represented by a power law, and that the structure noise can be linked to the parameters of the power spectra by the following relation:

$N \propto\left(\frac{d}{d_{0}}\right)^{1-\frac{\alpha}{2}} \times P_{0}^{\frac{1}{2}}$

where $d^{-1}$ is the spatial frequency, $d_{0}^{-1}$ is a reference spatial frequency, $P_{0}$ is the Fourier power at $d_{0}^{-1}$, and $\alpha$ is the spectral index of the Fourier power spectrum. It was also found that most scans can be described by the empirical relationships $\alpha \approx 3$ and $P_{0} \propto\langle B\rangle^{3}$.

Based on the IRAS results Helou \& Beichman (1990) (H\&B) proposed a practical formula to predict the cirrus confusion noise, assuming that the empirical relationships of Gautier et al. (1992) are valid at other wavelengths, too. They also took into account that at the resolution limit of the telescope $d / d_{0}$ can be expressed by the resolution parameter of Fraunhofer diffraction, $\lambda / D_{\mathrm{t}}$, where $\lambda$ is the wavelength of the measurement and $D_{\mathrm{t}}$ is the diameter of the telescope primary mirror. Under these terms they expressed the cirrus confusion noise as:

$\frac{N_{\mathrm{H \& B}}}{1 \mathrm{mJy}}=0.3 \times\left(\frac{\lambda}{100 \mu \mathrm{m}}\right)^{2.5}\left(\frac{D_{\mathrm{t}}}{1 \mathrm{~m}}\right)^{-2.5}\left(\frac{\left\langle B_{\lambda}\right\rangle}{1 \mathrm{MJy} \mathrm{sr}^{-1}}\right)^{1.5}$.

Here $B_{\lambda}$ is the surface brightness at the wavelength of the observation. The formula shows that the confusion noise depends on the wavelength in two separate ways: (1) via the variation of the surface brightness (spectral energy distribution of the emitting medium) and (2) via the dependence of the resolution parameter on $\lambda$. This relation which is valid for the standard measurement configuration as defined in Fig. 1 has been widely used for preparing far-infrared space telescope observations, and applied even for other configurations. The first noise analysis of four ISOPHOT maps was performed by Herbstmeier et al. (1998), carrying out the first high spatial resolution study of the galactic cirrus at $\lambda>100 \mu \mathrm{m}$. They proved that the ISOPHOT sensitivity at far-infrared wavelengths is limited by sky confusion noise rather than by instrumental uncertainties.

The confusion noise caused by the CFIRB has different properties from the cirrus confusion noise. It can be represented by a Poissonian spatial distribution, therefore the confusion noise value is independent of the separation between the target and the reference positions. Ackermann et al. (1992) investigated the sensitivity limits, including the effect of the CFIRB, for ISOPHOT and predicted that the long wavelength observations would be limited by galaxy confusion in the regions of faintest cirrus. Recently Lagache \& Puget (2000), Matsuhara et al. (2000) and Juvela et al. (for an overview see Lemke et al. 2000) analysed ISOPHOT maps and separated the cirrus and extragalactic components. They announced the detection of the CFIRB with a fluctuation power in the range of $5-12 \times 10^{3} \mathrm{Jy}^{2} \mathrm{sr}^{-1}$ at 170 and $180 \mu \mathrm{m}$, close to the predictions of Guiderdoni et al. (1997). This leads to a galaxy confusion noise limit of $13-22 \mathrm{mJy}$ for ISOPHOT at this wavelength.

For this paper we analysed 175 suitable far-infrared maps from the ISO Archive. Our goals were (1) to test the applicability of the Helou \& Beichman (1990) formula, especially at $\lambda>100 \mu \mathrm{m}$; (2) to determine the relative contributions of the galactic cirrus and the extragalactic components in the faintest regions of the sky; (3) to derive an easy-to-use formalism for predicting the total sky confusion noise for instruments on ISO, SIRTF and HERSCHEL and for various measurement configurations, and (4) to determine the Cosmic Far-Infared Background Radiation via its fluctuations.

\section{Observations and data analysis}

\subsection{Selection of ISOPHOT maps}

We selected 175 maps from the ISO Archive (Kessler et al. 2000), observed in the 90 to $200 \mu \mathrm{m}$ wavelength range, covering 40 different sky regions. All maps were obtained with the ISOPHOT instrument (Lemke et al. 1996) on-board the ISO satellite (Kessler et al. 1996), in the PHT22 raster observing mode (Laureijs et al. 2000). Our selection criteria were that the maps should be larger than $5 \times 5$ raster points, corresponding to $\sim 8^{\prime} \times 8^{\prime}$ for the $\mathrm{C} 100(3 \times 3$ pixel array, $46^{\prime \prime}$ pixel size) and C200 ( $2 \times 2$ pixel array, $92^{\prime \prime}$ pixel size) detectors. Some maps were observed with full oversampling, i.e. the field was redundantly covered without gaps between detector pixels. The final map images are a composite of the individual array images on the different raster positions. The size of the image pixels is always a detector pixel.

We excluded maps with obvious individual structures (resolved stars, galaxies, planetary nebulae, etc.). The number of selected maps is 94, 4, 65 and 12 using the C1_90, C1_100, C2_170 and C2_200 filters, respectively, and they cover a wide range of surface brightnesses $\left(\sim 1-100 \mathrm{MJysr}^{-1}\right)$. A more detailed description of the 
maps will be presented in a forthcoming paper (Kiss et al. 2001 , in prep.) dealing with the spatial structure of the extended emission.

\subsection{Data reduction}

Basic data reduction was performed using the ISOPHOT Interactive Analysis software, PIA V8.2 (Gabriel et al. 1997) with the standard batch mode set-up. Flat-fielding had to be applied, which corrects for the residual responsivity differences of the individual detector pixels. We chose the First Quartile Normalization method, which uses the first quartiles of the maps' brightness distribution, computed for each detector pixel, for normalization. The reliability of this statistical flat-field method was tested in the case of oversampled maps, were redundant observations of the same sky position by each detector pixel are available. These tests confirmed that the First Quartile Normalization gives accurate flat-field values, and was therefore adopted for this analysis.

The maps still contain the zodiacal light (ZL) emission, and a contribution from the CFRIB. The zodiacal emission does not contribute to the sky confusion noise, because its distribution is smooth on the scale of our maps (Ábrahám et al. 1997). Therefore, we can eliminate it from the total brightness. First, we determined the non color corrected ratio of the ZL-to-total emission at the DIRBE wavelengths, using the COBE/DIRBE weekly maps and the COBE/DIRBE sky and zodi atlas (Hauser et al. 1998; Kelsall et al. 1998). We colour corrected the ZL component adopting a spectral energy distribution (SED) shape of a black body with a temperature of $270 \mathrm{~K}$ (Leinert et al. 2001 find $255 \mathrm{~K} \leq T_{\mathrm{ZL}} \leq 297 \mathrm{~K}$ ). The difference between the uncorrected total and ZL emission was color corrected by assigning a cirrus SED to it, assuming a $20 \mathrm{~K}$ modified black body with $\nu^{2}$ emissivity law. Then interpolating the corrected ratios in between the COBE/DIRBE wavelengths, we estimated the ratios of the ZL-to-total emission for the ISOPHOT filter central wavelengths. The absolute ZL contribution in the ISOPHOT maps was computed by multiplying this ratio by the average total brightness measured by ISOPHOT (see also Héraudeau et al. 2001).

The remaining surface brightnesses include the CFIRB and the cirrus emission. In Sect. 3 we calculate the sky confusion noise and in Sect. 4.2 we separate the cirrus and Extragalactic Background fluctuations, and derive the value of the CFIRB.

\subsection{Instrument noise}

The determination of the instrument noise is crucial to get the real value of the sky confusion noise. In addition to the basic noise components (read-out noise, dark current variations, etc.), we also include in the instrument noise uncertainties related to the generation of the final maps (flat-field).
There are several ways to determine the instrument noise. The fundamental difference between them is the time scale over which the noise estimation was taken. We examined the following four methods:

- Ramp-noise: each measurement is composed of many individual integrations on each raster position. A set of non-destructive read-outs builds up an integration ramp whose slope provides the signal of this integration (Lemke et al. 1996). From each measurement we created two maps using the even and odd integration ramps separately. The instrument noise was calculated from the difference between the "even" and "odd" maps. This noise estimate provides information on the detector stability on a time scale of seconds, the typical difference between adjacent ramps;

- PIA-noise: PIA provides a determination of the signal uncertainty of the individual raster positions, which can also be adopted as an estimate of $N_{\text {inst }}$. It reflects the error propagation of random error components in (1) the linear fitting of the integration ramps, and (2) the averaging of all signals per raster point ${ }^{1}$. The time scale here is the time spent on an individual raster position, typically in the order of a minute;

- Flat-field-noise: this noise value was calculated from the variation of the brightness at the same sky position in maps created from individual detector pixels (i.e. in case of pixel redundancy), after correction by the appropriate flat-fielding factors. Since these factors are kept constant for the whole map, although the detector pixels show different time dependent transient curves, this instrument noise estimate includes the effects of the long-term changes in the detector behaviour. Therefore the time scale is the observational time of the whole map, typically in the order of an hour. Since the transient behaviour depends on the illumination level, the flat-field-noise may also depend on the brightness of the map;

- Repetition-noise: if a sky region has been observed several times, then it is possible to derive a noise value from the comparison of the individual maps. The typical time scale here is the difference in the observation dates. It provides a measure of the measurement reproducibility.

The ramp- and PIA-noise could be calculated for each map, but the flat-field-noise could be evaluated only in maps with full oversampling (see Sect. 2.1) and the repetition-noise was only computed for one particular sky region (Marano 1 field) consisting of several submaps, each observed four times. A comparison of the different noise estimates showed that the ramp-noise provided the lowest $N_{\text {inst }}$ values, typically a factor of 2 lower than the PIAnoise. The flat-field noise was higher than the PIA one, and the repetition noise was found to be nearly identical to the flat-field-noise. Since in the case of the structure

\footnotetext{
1 The on-line PIA-manual is available at the URL: http://www.iso.vilspa.esa.es/manuals/PHT/pia/pia.html
} 


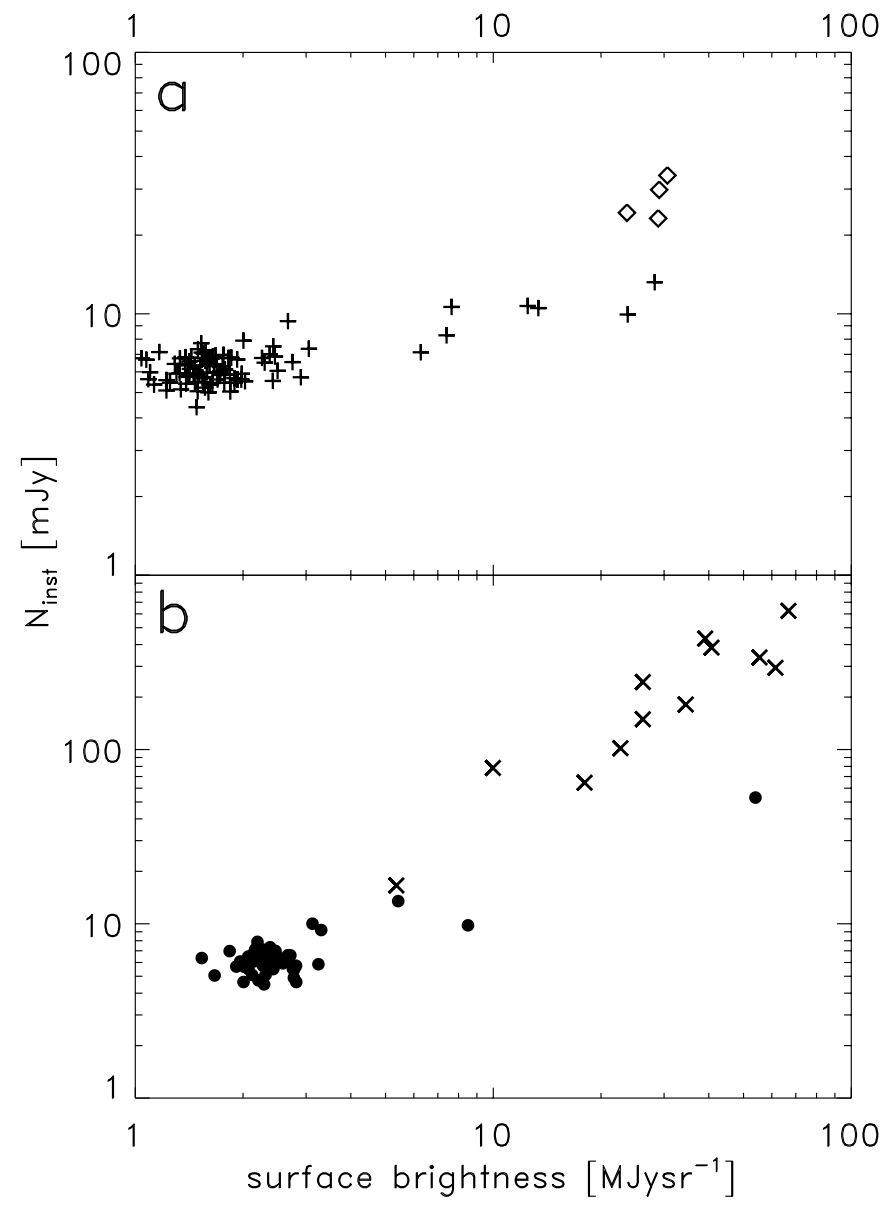

Fig. 2. Instrument noise values measured on the ISOPHOT maps. In the case of oversampled maps the flat-field noise, otherwise the scaled up PIA-noise was adopted. The mean scaling factor between the PIA- and the flat-field noise values are 1.35 and 1.65 for the C_100 and C_200 detectors, respectively. a) C100 detector: C1_90 and C1_100 filters (plus signs and diamonds, respectively), b) C200 detector: C2_170 and C2_200 filters (black dots and crosses, respectively).

noise calculation (Eq. (1)) the target and reference positions were observed at times typically separated by several minutes, the correct instrument noise should lie between the PIA- and the flat-field-noise values. To be on the safe side, we chose the value of the flat-field-noise, although it may represent a somewhat conservative estimate, which might cause a slight underestimation in the final confusion noise values. Since this noise value is not available for each map, we took the uncertainties provided by PIA and scaled them according to the mean ratio of 1.35 and 1.65 of the flat-field- and PIA-noise values for the C100 and C200 detectors, respectively.

The typical instrument noise values we found (see Fig. 2) are somewhat higher than that of other authors, e.g. a factor of 2 compared to Lagache et al. (2000). This discrepancy can be traced back to the co-addition of four independent maps in their case, reducing the final noise values by this factor.

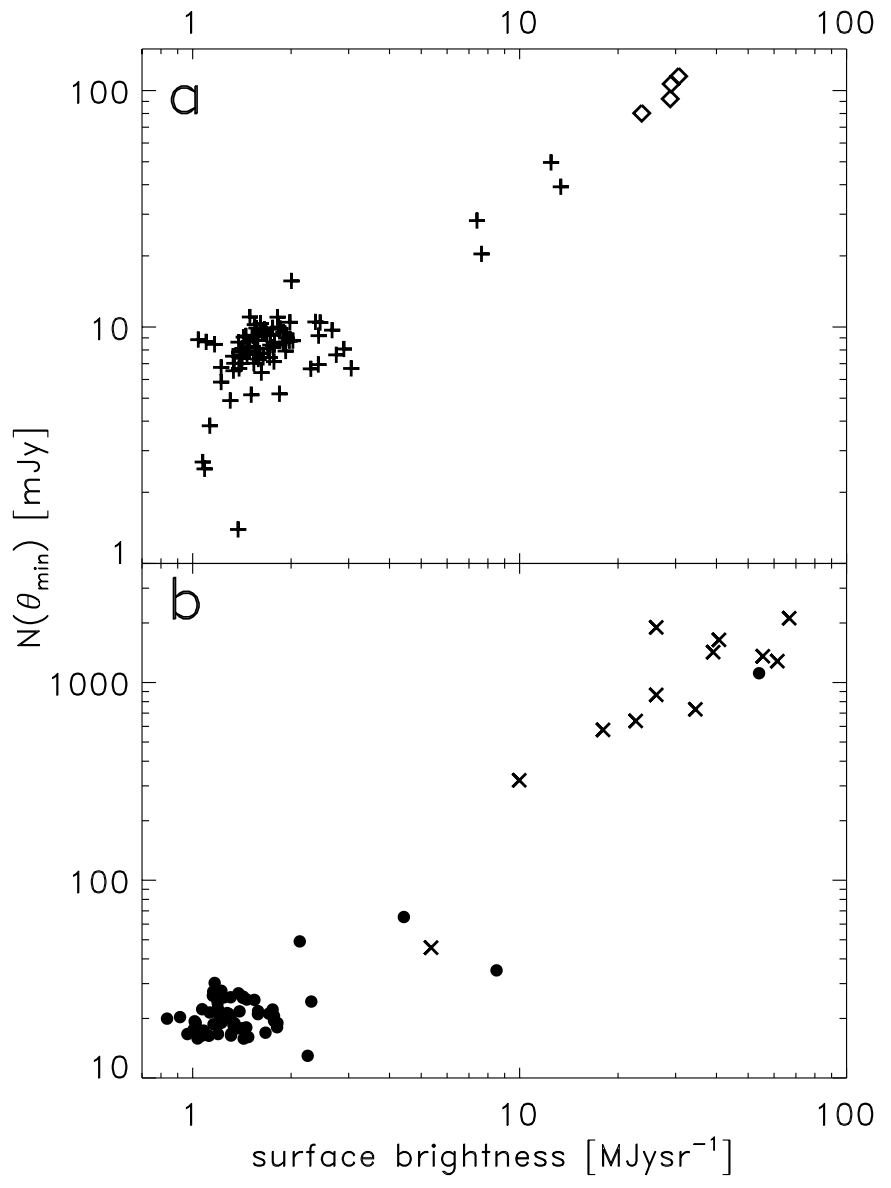

Fig. 3. Confusion noise at the resolution limit $\theta_{\min }$ vs. surface brightness of the fields. a) ISOPHOT C100 filters, C1_90 and C1_100. b) ISOPHOT C200 filters, C2_170 and C2_200. The symbols are the same as in Fig. 2.

Following Herbstmeier et al. (1998) we assumed that the sky brightness fluctuations and instrument noise contributions are statistically independent, therefore the measured structure noise can be expressed as:

$N_{\text {meas }}^{2} \leq N^{2}+2 \cdot N_{\text {inst }}^{2}$

where $N_{\text {meas }}$ is the measured structure noise, $N$ is the real structure noise and $N_{\text {inst }}$ is the instrument noise. We used this formula for the subtraction of the instrument noise from the measured noise, by assuming equality between the two sides of the expression. In order to test this assumption we compared the confusion noise values of four C2_170 maps, where the same sky region was mapped repeatedly. The confusion noise must be the same and only the instrument noise values can differ in repeated observations. Assuming equality in Eq. (5) we obtained identical confusion noise values for all four measurements and for any separations. 
Table 1. Results of the fits using the measured values of the sky confusion noise at the resolution limit $N\left(\theta_{\min }\right)$ and the average surface brightness of the field $\langle B\rangle$ (see also Eq. (6) and the text for details). Separation limit and effective solid angle values are also presented. In the case of the C1_100 and C2_200 filters no appropriate determination of the $C_{0}$ offset was possible due to the lack of faint regions.

\begin{tabular}{ccccc}
\hline filter & C1_90 & C1_100 & C2_170 & C2_200 \\
\hline$C_{0}$ & $6.6 \pm 1.9$ & - & $10.2 \pm 2.5$ & - \\
$C_{1}$ & $0.7 \pm 0.3$ & $0.6 \pm 0.1$ & $3.0 \pm 0.3$ & $4.4 \pm 0.2$ \\
$\eta$ & $1.55 \pm 0.12$ & $1.53 \pm 0.28$ & $1.47 \pm 0.11$ & $1.56 \pm 0.19$ \\
$\theta_{\min }$ & $92^{\prime \prime}$ & $92^{\prime \prime}$ & $184^{\prime \prime}$ & $184^{\prime \prime}$ \\
$\Omega\left(10^{-7} \mathrm{sr}\right)$ & 0.6469 & 0.7030 & 2.6438 & 2.8120 \\
\hline
\end{tabular}

\section{Results}

\subsection{Confusion noise at the resolution limit}

Figure 3 presents our results on the sky confusion noise (after removing the instrument noise using Eq. (5)) measured with the four ISOPHOT filters C1_90, C1_100, C2_170 and C2_200. We used the convention $\theta=2 D$, defined by Gautier et al. (1992) (see Sect. 1). Following the H\&B formula (Eq. (4)) we fitted the data points for each filter separately, assuming a power-law relationship between the confusion noise and the average brightness of the field, but also allowing a constant factor:

$\frac{N\left(\theta_{\mathrm{min}}\right)}{1 \mathrm{mJy}}=C_{0}+C_{1} \times\left(\frac{\langle B\rangle}{1 \mathrm{MJy} \mathrm{sr}^{-1}}\right)^{\eta}$.

The coefficients $C_{0}, C_{1}$ and $\eta$ are listed in Table 1 . In the case of the C1_100 and C2_200 filters no appropriate determination of the $\mathrm{C}_{0}$ offset was possible due to the lack of faint regions. Equation (6) can be used to predict the confusion noise for the ISOPHOT filters studied here. Our confusion noise values measured at $170 \mu \mathrm{m}$ are in agreement with a value of 45 mJy obtained by Dole et al. (2001) in the FIRBACK regions, taking into account that their value is a source flux confusion limit, which differs from ours by the footprint-fraction and is therefore a factor of 2 higher.

\subsection{Comparison with the $H \& B$ formula}

In Fig. 4 we compare our results with the predictions of the $\mathrm{H} \& \mathrm{~B}$ formula (Eq. (4)). In the surface brightness range where the cirrus emission dominates (5-30 $\mathrm{MJy} \mathrm{sr}^{-1}$ ) the $\mathrm{H} \& \mathrm{~B}$ formula predicts confusion noise values within a factor of 2 of our results. The scatter cannot be attributed to measurement uncertainties, and probably it reflects the physical differences among the fields. For the brightest fields the $\mathrm{H} \& \mathrm{~B}$ formula seems to systematically overestimate the measured confusion noise values. Such regions, however, contain molecular clouds, therefore they may have different spatial structure from a cirrus region and should be described by a different power law. The figure also shows a large discrepancy at low surface brightness

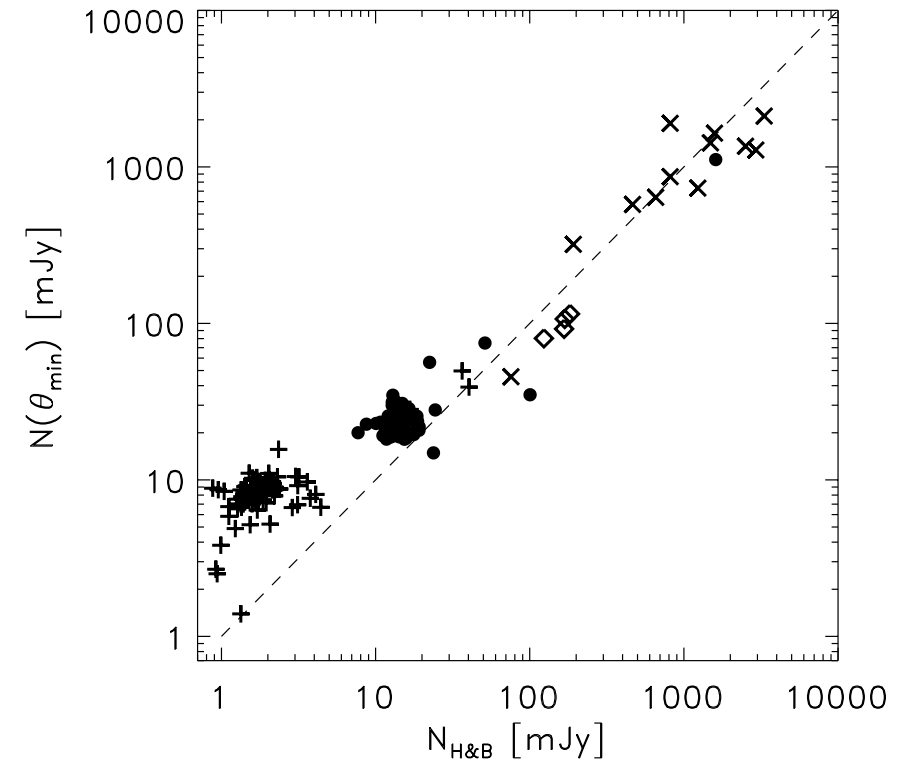

Fig. 4. Confusion noise at the resolution limit determined by this study as compared to the prediction of Helou \& Beichman (1990). The symbols are the same as in Fig. 2.

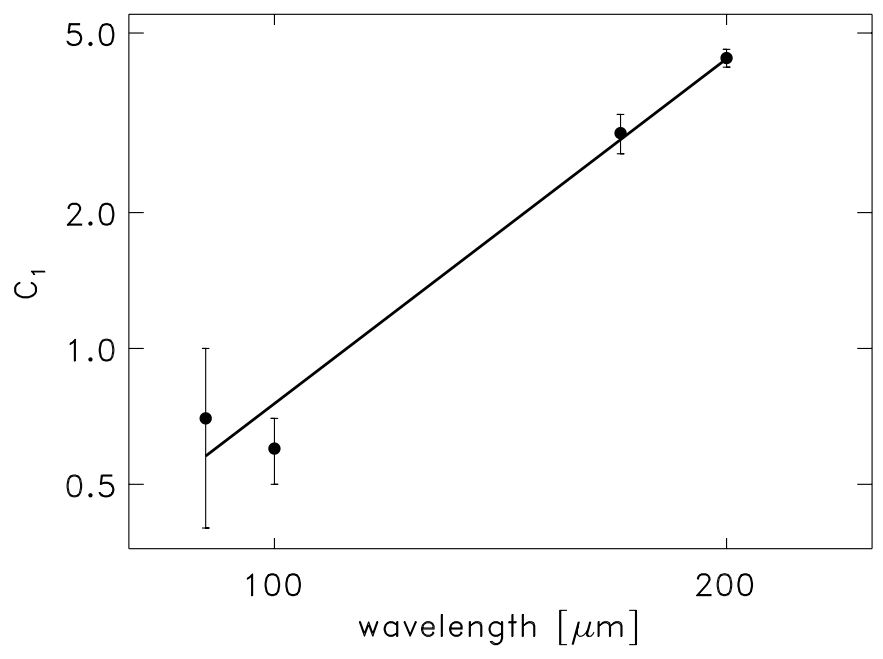

Fig. 5. $C_{1}$ coefficients of the fits in Table 1 vs. the appropriate wavelength (logarithmic-logarithmic plot).

which could well be treated by the introduction of an offset (see above). A possible explanation of the deviations in the faint range is discussed in Sect. 4.2.

Although the H\&B formula gives rather good predictions for the surface brightness range it was developed for $\left(5-30 \mathrm{MJysr}^{-1}\right)$, its coefficients, especially the three exponents, rely partly on the analysis of IRAS $100 \mu \mathrm{m}$ data, partly on theoretical considerations. Our data set offers a unique chance to test some of these exponents for the first time. The dependence of the confusion noise on the surface brightness was described by $\mathrm{H} \& \mathrm{~B}$ using a power law with an exponent of 1.5 (Eq. (4)), independently of the wavelength. This exponent $\eta$ was determined by our fits for four wavelengths in Sect. 3.1. The measured values 
in Table 1 are very close to the value of 1.5 , proving the wavelength independence of this exponent.

In the next step we tested the dependence of the confusion noise on the resolution parameter $\lambda / D_{\mathrm{t}}$, also assumed to be in the form of a power law with an exponent of 2.5 by H\&B (Eq. (4)). Since the diameter of the telescope mirror $D_{\mathrm{t}}$ is the same regardless of the wavelength, in Fig. 5 we plotted the wavelength dependence of the $C_{1}$ coefficient from Table 1. The figure confirms the assumption of a power law, and gives an exponent of $2.53 \pm 0.31$, in agreement with the value in Eq. (4). The dependence of the confusion noise on the diameter of the telescope (Eq. (4)) was not possible to check, since the telescope mirror of ISO had the same size as that of IRAS. Our results verify exponents of the $\mathrm{H} \& \mathrm{~B}$ formula for the first time by observations over a wide range.

\subsection{Confusion noise for larger separations}

The H\&B formula provides information only on the measurement configuration presented in Fig. 1, at the resolution limit $\theta_{\min }$. However, in many applications the beam separation may be different. In most ISOPHOT measurements the observer was allowed to specify any separation up to $3^{\prime}$. Staring on-off measurements and sparse maps allowed even larger separations (Laureijs et al. 2000). Properties of the sky confusion noise for these cases were not investigated so far.

As indicated in Sect. 1, the ratio of the confusion noise values measured at different separations is sensitive to the spatial distribution of the dominant component of the emission (galactic cirrus or distant galaxies). We found that the dependence of the sky confusion noise on the separation between target and reference sky positions can be described by a simple power law:

$N\left(q \cdot \theta_{\min }\right)=N\left(\theta_{\min }\right) \times q^{\gamma}$

where $q=1,1 \frac{1}{2} \ldots 3 \frac{1}{2}$ and $\gamma$ is constant for a specific map. We fitted $\gamma$ for all maps. The results are presented in Fig. 6, which suggests that the $\gamma$ value is a filterindependent parameter of the field, depending only on the surface brightness. For faint fields $\gamma$ is close to zero, while it increases strongly with increasing surface brightness. We found that the behaviour can be approximated by the following relation (dotted curve in Fig. 6):

$\gamma= \begin{cases}0.02 & \text { if }\langle B\rangle \leq 3 \mathrm{MJy} \mathrm{sr}^{-1} \\ 0.65 \times \log _{10}\langle B\rangle-0.26 & \text { if }\langle B\rangle>3 \mathrm{MJy} \mathrm{sr}^{-1}\end{cases}$

Equations (7) and (8) together with Eq. (6) and the coefficients in Table 1 provide a practical tool for the estimation of the confusion noise for a large variety of measurements.

\section{Discussion}

\subsection{Variations of $\gamma$}

As shown in Sect. 3.3, for low surface brightness fields the $\gamma$ values are close to zero. In those regions the noise

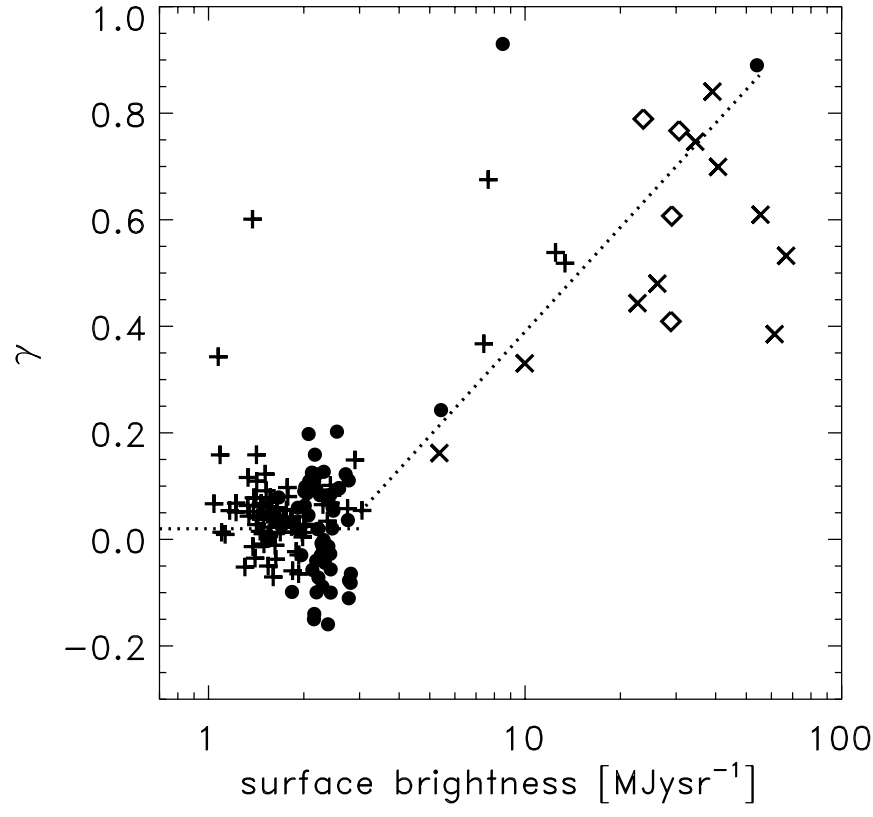

Fig. 6. $\gamma$ values of the $N\left(q \cdot \theta_{\min }\right)=N\left(\theta_{\min }\right) \cdot q^{\gamma}$ fits for four ISOPHOT filters versus the surface brightness of the fields (see the text for details). The symbols are the same as in Fig. 2.

distribution differs from that expected for the galactic cirrus (see e.g. Ackermann et al. 1992), originating from its multifractal structure. $\gamma \approx 0$ is typical for a Poissonian distribution.

Higher $\gamma$ values in brighter regions may be caused by the cirrus structure. Despite this general trend the scatter of $\gamma$ at higher surface brightness is relatively large and there are a few regions measured by the C1_90 filter with high $\gamma$ values at low brightness. These discrepancies are probably caused by real physical differences between the fields. The $\gamma$ parameter depends on the spectral index of the Fourier power spectrum of the field (cf. Eq. (3)). This relationship will be analysed in detail based on experimental data in a forthcoming paper (Kiss et al. 2001, in prep.). Differences in physical properties may originate in different chemical/dust composition, spatial structure, gas-to-dust or molecular-to-neutral gas ratio.

\subsection{Fluctuations due to the extragalactic background}

\subsubsection{Findings from this work}

When fitting the sky confusion noise as a function of the surface brightness in Sect. 3.1 a constant term $C_{0}$ was allowed for. At both 90 and $170 \mu \mathrm{m}$ definite positive values were obtained at the $3-4 \sigma$ significance level. Assuming that the cirrus confusion noise follows the power law behaviour also at very low surface brightness, this offset cannot be attributed to cirrus. The spatial distribution of the confusion noise below $\sim 3 \mathrm{MJysr}^{-1}$ has different characteristics than in brighter cirrus fields (see Sect. 3.3 and Fig. 6). The likely origin of this noise component, as suggested by its invariability with the surface brightness and 
its Poissonian spatial distribution, would be the fluctuation due to the Cosmic Far-Infrared Background (CFIRB).

If this interpretation is correct, than Eq. (6) provides a new method to determine the value of the CFIRB fluctuation. The features of this method are (1) the subtraction of the cirrus component via its dependence on the surface brightness and (2) that this dependence is calibrated simultaneously on the brighter cirrus fields of the same database. An advantage of this method is the utilization of the largest ISOPHOT database for the determination of the CFIRB fluctuations. The results are not sensitive to characteristics in individual fields. On the other hand, any uncertainty in the surface brightness calibration of the cirrus would introduce an uncertainty in the fluctuation value, too.

The values given by the fitting procedure in Sect. 3.1 for the $C_{0}$ coefficients are $6.6 \pm 1.9$ and $10.2 \pm 2.5 \mathrm{mJy}$ at 90 and $170 \mu \mathrm{m}$, respectively. In this fitting the surface brightness values still included a contribution from the CFIRB itself. Subtraction of the CFIRB increases the fluctuation amplitudes. Varying the CFIRB surface brightness $B_{0}$ within the assumed range of 0.1 to $1.5 \mathrm{MJy} \mathrm{sr}^{-1}$ and subtracting it from the total surface brightness we used Eq. (6) to derive the corresponding $C_{0}$ coefficients which were then transferred to surface brightness fluctuations $\delta B_{0}$ as $\delta B_{0}=C_{0} / \Omega_{\lambda}$, where $\Omega_{\lambda}$ is the effective solid angle of a detector pixel (see Table 1 ).

The results are shown in Fig. 7. As can be seen in this figure, the dependence of $\delta B_{0}$ on the assumed value of $B_{0}$ is not very strong, therefore, regardless of the real value of the CFIRB, a well confined value of $\delta B_{0}$ can be derived. We obtained $C_{0}=7 \pm 2 \mathrm{mJy}\left(\delta B_{0}=0.11 \pm\right.$ $\left.0.03 \mathrm{MJy} \mathrm{sr}^{-1}\right)$ at $90 \mu \mathrm{m}$ and $C_{0}=15 \pm 4 \mathrm{mJy}\left(\delta B_{0}=\right.$ $0.06 \pm 0.02 \mathrm{MJy} \mathrm{sr}^{-1}$ ) at $170 \mu \mathrm{m}$.

In most models CFIRB fluctuations are caused by galaxy clustering, and, due to the flat power spectrum, the $\delta B_{0} / B_{0}$ value is constant in the $1^{\circ}$ to $5^{\prime}$ resolution range (Kashlinsky et al. 1996; Haiman \& Knox 2000). At finer resolution (higher spatial frequencies) effects by individual galaxies have to be considered. An estimate of $\delta B_{0} / B_{0}$ (or $\left.\delta\left(\nu I_{\nu}\right) /\left(\nu I_{\nu}\right)\right)$ can be performed following Bond et al. (1986), assuming biased galaxy clustering. Using Eq. (6.35) from Bond et al. (1986), the fluctuation ratio is: $\delta\left(\nu I_{\nu}\right) /\left(\nu I_{\nu}\right)=0.05 \times\left(1^{\prime} / \sigma\right)^{0.4}$ where $\sigma$ is the smoothing angle, and the beam profile is approximated by a Gaussian. Equations (6.24) and (7.2) in Bond et al. (1986) give $\sigma=12^{\prime \prime}$ and $23^{\prime \prime}$ for 90 and $170 \mu \mathrm{m}$, respectively, for ISOPHOT. From this results $\delta\left(\nu I_{\nu}\right) /\left(\nu I_{\nu}\right)=10 \%$ at $90 \mu \mathrm{m}$ and $\delta\left(\nu I_{\nu}\right) /\left(\nu I_{\nu}\right)=7 \%$ at $170 \mu \mathrm{m}$.

At $170 \mu \mathrm{m} \delta B_{0} / B_{0}=7 \%$ provides $B_{0}=0.8 \pm$ $0.2 \mathrm{MJy} \mathrm{sr}^{-1}$ equivalent to $\nu I_{\nu}=14 \pm 3 \mathrm{nW} \mathrm{m}^{-2} \mathrm{sr}^{-1}$.

At $90 \mu \mathrm{m}$ the dependence of $\delta B_{0}$ on $B_{0}$ is even weaker than at $170 \mu \mathrm{m}$, therefore a well defined value of $0.11 \pm$ $0.03 \mathrm{MJy} \mathrm{sr}^{-1}$ can be determined. The $B_{0}$ value of $1.1 \pm$ $0.3 \mathrm{MJy} \mathrm{sr}^{-1}$, equivalent to $\nu I_{\nu}=37 \pm 10 \mathrm{nW} \mathrm{m}^{-2} \mathrm{sr}^{-1}$ ), is the same as the average surface brightness of the faintest $90 \mu \mathrm{m}$ maps in our data set. The expected cirrus

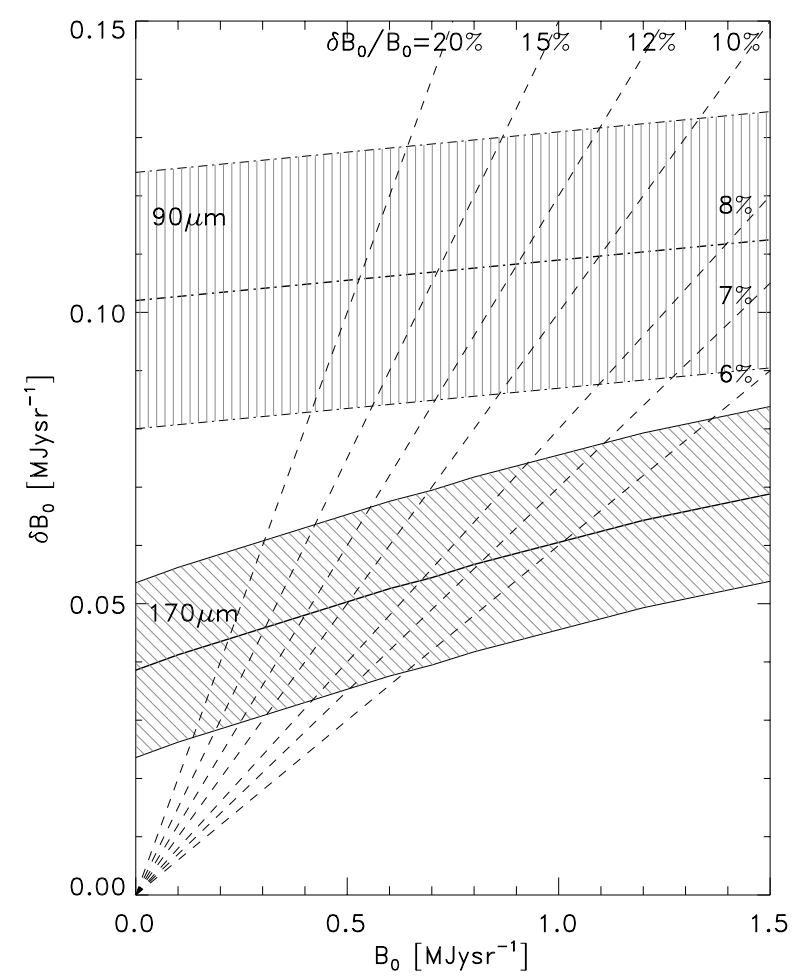

Fig. 7. CFIRB fluctuation amplitudes versus the CFIRB absolute level assumed. The hatched regions represent the formal errors of the fits in Eq. (6) for 90 and $170 \mu \mathrm{m}$. A set of dashed lines marks $\delta B_{0} / B_{0}=6,7,8,10,12,15$ and $20 \%$.

contribution in these fields - predicted from the $\sim 0.8 \mathrm{MJysr}^{-1}$ value at $170 \mu \mathrm{m}$ by using a typical cirrus spectrum (modified black-body SED, $\nu^{2}$ emissivity law, $20 \mathrm{~K}$ temperature) - is about $0.3 \mathrm{MJy} \mathrm{sr}^{-1}$. This result suggests that the $1.1 \mathrm{MJysr}^{-1}$ is an upper limit and the most likely value of the CFIRB lies at the lower end of the uncertainty range, i.e. $\sim 0.8 \mathrm{MJy} \mathrm{sr}^{-1}$ equivalent to $\nu I_{\nu}=$ $30 \mathrm{nW} \mathrm{m}^{-2} \mathrm{sr}^{-1}$.

Our results satisfy the three main criteria of a CFIRB detection as proposed by Hauser \& Dwek (2001): (1) We achieved a detection of a positive value of the fluctuation amplitudes (and therefore the absolute value as well) at 90 and $170 \mu \mathrm{m}$ at the $4 \sigma$ level. (2) We removed the contribution by all known foreground (noise) emitters (zodiacal light, instrument noise, galactic cirrus). The properties of the spatial distribution related to the remaining noise term agree with that expected for the CFIRB fluctuations. (3) Due to the statistical nature of our method, the detection of the same well-defined positive constant term in different areas of the far-infrared sky indicates the isotropy of this component.

\subsubsection{Comparison with other results}

The $170 \mu \mathrm{m}$ confusion noise $C_{0}=15 \pm 4 \mathrm{mJy}$ is in good agreement with the $13 \mathrm{mJy}$ obtained by Juvela et al. (see the overview of Lemke et al. 2000), and somewhat lower than the values of 18 mJy determined by Lagache \& Puget (2000) from the analysis of the Marano 1 field and the 
22 mJy derived by Matsuhara et al. (2000) from the investigation of the Lockman Hole region. Our derived CFIRB value of $14 \pm 3 \mathrm{nW} \mathrm{m}^{-2} \mathrm{sr}^{-1}$ is the same as predicted from models by Pei et al. (1999). Also the COBE measurements calibrated on the FIRAS photometric scale (Hauser et al. 1998 for an overview see Hauser \& Dwek 2001) give quite similar levels $\left(15 \pm 6 \mathrm{nW} \mathrm{m}^{-2} \mathrm{sr}^{-1}\right.$ at $140 \mu \mathrm{m}$ and $13 \pm 2 \mathrm{nW} \mathrm{m}^{-2} \mathrm{sr}^{-1}$ at $\left.240 \mu \mathrm{m}\right)$ despite the big difference in spatial resolution.

The $90 \mu \mathrm{m}$ confusion noise $C_{0}=7 \pm 2 \mathrm{mJy}$ is close (although somewhat lower) to the $11 \mathrm{mJy}$ found by Matsuhara et al. (2000). With regard to the $90 \mu \mathrm{m}$ absolute value Schlegel et al. (1998) provided the same $1.1 \mathrm{MJysr}^{-1}$ value as an upper limit for the CFIRB at $100 \mu \mathrm{m}$, and also Lagache et al. (2000) and Finkbeiner et al. (2000) reported a detection of a $100 \mu \mathrm{m}$ CFIRB level of $23 \pm 6 \mathrm{nW} \mathrm{m}^{-2} \mathrm{sr}^{-1}$ and $25 \pm 8 \mathrm{nW} \mathrm{m}^{-2} \mathrm{sr}^{-1}$, respectively, which are close to our upper limit.

\subsection{Confusion limits for far-infrared space telescopes}

After the comparison of instrument and confusion noise values (see Figs. 2 and 3 ) it is obvious that even in the faintest sky regions the instrument noise is below the sky confusion noise by a factor of $2-3$ for the C2_170 filter. This confirms the results of Herbstmeier et al. (1998), showing that the ISOPHOT C200 detector was limited by sky brightness fluctuations rather than by instrumental effects. In the case of the C1_90 filter the instrumental and confusion noise are similar in strength at the resolution limit. Since our results confirm the applicability of the $\mathrm{H} \& \mathrm{~B}$ formula, the predictions made for other space missions with telescopes of a similar size (SIRTF, Astro-F) on the basis of this formula can be trusted. Predictions made for the HERSCHEL satellite (3.6 m mirror), however, still have to partially rely on assumptions, since we were not able to test the dependence of the confusion noise on the diameter of the telescope primary mirror. It remains to be demonstrated that the spatial structure of cirrus does not change below the resolution limit of ISO.

\section{Summary}

Using measurements obtained with ISOPHOT we investigated the properties of the sky confusion noise on a large sample of maps in the $90 \leq \lambda \leq 200 \mu \mathrm{m}$ wavelength range. We described the dependence of the sky confusion noise on the surface brightness for four selected ISOPHOT filters. We verified that the confusion noise scales as $N \propto\langle B\rangle^{1.5}$ for the resolution limit, independent of the wavelength. We also confirmed that, due to the dependence on the resolution parameter, $N \propto \lambda^{2.5}$ at $\lambda \geq 100 \mu \mathrm{m}$. According to our results for cirrus fields with $\langle B\rangle>5 \mathrm{MJy} \mathrm{sr}^{-1}$ the Helou \& Beichman (1990) formula predicts confusion noise values within a factor of 2 . The scaling of the noise value at different separations between target and reference positions was investigated for the first time, providing a useful formula to estimate the confusion for different separations, too. At 90 and $170 \mu \mathrm{m}$ a noise term with a Poissonian spatial distribution was detected in the faintest fields $\left(\langle B\rangle \leq 3-5 \mathrm{MJy} \mathrm{sr}^{-1}\right)$, which we interpret as fluctuations in the Cosmic Far-Infared Background (CFIRB). With a ratio of the fluctuation amplitude to the absolute level of $10 \%$ and $7 \%$ at 90 and $170 \mu \mathrm{m}$, respecively, we determined the fluctuation amplitudes and the surface brightness of the CFIRB. The fluctuation amplitudes are $7 \pm 2 \mathrm{mJy}$ and $15 \pm 4 \mathrm{mJy}$ at 90 and $170 \mu \mathrm{m}$, respectively. We obtained a CFIRB surface brightness of $B_{0}=0.8 \pm 0.2 \mathrm{MJysr}^{-1}\left(\nu I_{\nu}=14 \pm 3 \mathrm{nW} \mathrm{m}^{-2} \mathrm{sr}^{-1}\right)$ at $170 \mu \mathrm{m}$ and an upper limit of $1.1 \mathrm{MJysr}^{-1}\left(\nu I_{\nu}=\right.$ $37 \mathrm{nW} \mathrm{m}^{-2} \mathrm{sr}^{-1}$ ) at $90 \mu \mathrm{m}$.

Acknowledgements. The development and operation of ISOPHOT were supported by MPIA and funds from Deutsches Zentrum für Luft- und Raumfahrt (DLR, formerly DARA). The ISOPHOT Data Center at MPIA is supported by Deutsches Zentrum für Luft- und Raumfahrt e.V. (DLR) with funds of Bundesministerium für Bildung und Forschung, grant No. 50 QI 98013 . This research was partly supported by the ESA PRODEX programme (No. 14594/00/NL/SFe) and by the Hungarian Research Fund (OTKA F-022566). M.J. acknowledges the support of the Academy of Finland Grant No. 1011055. The authors are responsible for the contents of this publication.

\section{References}

Ábrahám, P., Leinert, Ch., \& Lemke, D. 1997, A\&A, 328, 702 Ackermann, E., Hajduk, C., \& Lemke, D. 1992, Sensitivity Limits for the Photopolarimeter ISOPHOT, in Infrared Astronomy with ISO, ed. Th. Encrenaz, \& M. F. Kessler (Nova Science Publisher), 79

Bond, J. R., Carr, B. J., \& Hogan, C. J. 1986, ApJ, 428, 306

Dole, H., Gispert, R., Lagache, G., et al. 2001, A\&A, 372, 364

Finkbeiner, D. P., Davis, M., \& Schlegel, D. J. 2000, ApJ, 544,81

Gabriel, C., Acosta-Pulido, J., Heinrichsen, I., et al. 1997, The ISOPHOT Interactive Analysis PIA, a Calibration and Scientific Analysis Tool, in ADASS VI, ed. G. Hunt, \& H. E. Payne, ASP Conf. Ser., 125, 108

Gautier III, T. N., Boulanger, F., Pérault, M., \& Puget, J. L. 1992, AJ, 103, 1313

Guiderdoni, B., Bouchet, F. R., Puget, J. L., Lagache, G., \& Hivon, E. 1997, Nature, 390, 257

Haiman, Z., \& Knox, L. 2000, ApJ, 530, 124

Hauser, M. G., Arendt, R. G., Kessel, T., et al. 1998, ApJ, 508,25

Hauser, M. G., \& Dwek, E., ARA\&A, in press [ASTRO-PH 0105539]

Helou, G., \& Beichman, C. A. 1990, The confusion limits to the sensitivity of submilimeter telescopes, in From GroundBased to Space-Borne Sub-mm Astronomy, Proc. of the 29th Liège International Astrophysical Coll. (ESA Publ.), 117 (H\&B)

Héraudeau, Ph., Ábrahám, P., Klaas, U., Kiss, Cs., \& del Burgo, C., Systematic comparison of ISOPHOT and DIRBE Surface Brightness Calibration, in The calibration legacy of the ISO Mission, ed. L. Metcalfe et al., ESA Spec. Publ. Ser., 481, in press 
Herbstmeier, U., Ábrahám, P., Lemke, D., et al. 1998, A\&A, 332,739

Kashlinsky, A., Mather, J. C., \& Odenwald, S. 1996, ApJ, 473, L9

Kelsall, T., Weiland, J. L., Franz, B. A., et al. 1998, ApJ, 508, 44

Kessler, M. F., Steinz, J. A., Anderegg, M. F., et al. 1996, A\&A, 315, 27

Kessler, M. F., Müller, T. G., Arviset, C., García-Lario, P., \& Prusti, T. 2000, The ISO Handbook, vol. I, ISO - Mission Overview, SAI-2000-035/Dc, Version 1.0, ISO Data Centre, Villafranca del Castillo

Lagache, G., Abergel, A., Boulanger, F., Désert, F. X., \& Puget, J.-L. 1999, A\&A, 344, 322

Lagache, G., \& Puget, J.-L. 2000, A\&A, 355, 17

Lagache, G., Haffner, L. M., Reynolds, R. J., \& Tufte, S. L. 2000, A\&A, 354, 247

Laureijs, R. J., Klaas, U., Richards, P. J., Schulz, B., \& Ábrahám, P. 2000, The ISO Handbook, vol. V, PHT
- The Imaging Photo-Polarimeter, SAI/99-069/Dc, Version 1.2, ISO Data Centre, Villafranca del Castillo

Leinert, Ch., Ábrahám, P., Acosta-Pulido, J., Lemke, D., \& Siebenmorgen, R. 2001, A\&A, submitted

Lemke, D., Klaas, U., Abolins, J., et al. 1996, A\&A, 315, 64

Lemke, D., Ábrahám, P., Haas, M., et al. 2000, ISOPHOT Surveys and the Extragalactic Background, in The Extragalactic Background and its Cosmological Implications, IAU Symp. 204, ed. M. Harwit, \& M. G. Hauser, in press

Low, F., Beintema, D. A., Gautier, T. N., et al. 1984, ApJ, 278,19

Matsuhara, H., Kawara, K., Sato, Y., et al. 2000, A\&A, 361, 407

Pei, Y. C., Fall, M. S., \& Hauser, M. G. 1999, ApJ, 522, 604

Schlegel, D. J., Finkbeiner, D. P., \& Davis, M. 1998, ApJ, 500, 525 